\title{
A Modular Dual-Labeling Scaffold That Retains Agonistic Properties for Somatostatin Receptor Targeting
}

\author{
Sukhen C. Ghosh ${ }^{1}$, Melissa Rodriguez ${ }^{2}$, Kendra S. Carmon ${ }^{1}$, Julie Voss ${ }^{1}$, Nathaniel L. Wilganowski ${ }^{1}$, \\ Agnes Schonbrunn ${ }^{2}$, and Ali Azhdarinia ${ }^{1}$ \\ ${ }^{I}$ The Brown Foundation Institute of Molecular Medicine, McGovern Medical School, The University of Texas Health Science Center \\ at Houston, Houston, Texas; and ${ }^{2}$ Department of Integrative Biology and Pharmacology, McGovern Medical School, The University \\ of Texas Health Science Center at Houston, Houston, Texas
}

Fluorescence-guided surgery is an emerging imaging technique that can enhance the ability of surgeons to detect tumors when compared with visual observation. To facilitate characterization, fluorescently labeled probes have been dual-labeled with a radionuclide to enable cross-validation with nuclear imaging. In this study, we selected the somatostatin receptor imaging agent DOTATOC as the foundation for developing a dual-labeled analog. We hypothesized that a customized dual-labeling approach with a multimodality chelation (MMC) scaffold would minimize steric effects of dye conjugation and retain agonist properties. Methods: An MMC conjugate (MMC-TOC) was synthesized on solid-phase and compared with an analog prepared using conventional methods (DA-TOC). Both analogs were conjugated to IRDye 800 using copper-free click chemistry. The resulting compounds, MMC(IR800)-TOC and DA(IR800)-TOC, were labeled with $\mathrm{Cu}$ and ${ }^{64} \mathrm{Cu}$ and tested in vitro in somatostatin receptor subtype 2-overexpressing HEK-293 cells to assess agonist properties, and in AR42J rat pancreatic cancer cells to determine receptor binding characteristics. Multimodality imaging was performed in AR42J xenografts. Results: Cu-MMC(IR800)-TOC demonstrated higher potency for cyclic adenosine monophosphate inhibition (half maximal effective concentration $\left[E_{50}\right]$ : $0.21 \pm 0.18$ vs. $1.38 \pm 0.54 \mathrm{nM})$ and receptor internalization $\left(\mathrm{EC}_{50}: 41.9 \pm 29.8\right.$ vs. $455 \pm 299 \mathrm{nM})$ than Cu-DA(IR800)-TOC. Radioactive uptake studies showed that blocking with octreotide caused a dosedependent reduction in ${ }^{64} \mathrm{Cu}-\mathrm{MMC}(\mathrm{IR} 800)$-TOC uptake whereas ${ }^{64} \mathrm{Cu}-\mathrm{DA}(\mathrm{IR} 800)-\mathrm{TOC}$ was not affected. In vivo studies revealed higher tumor uptake for ${ }^{64} \mathrm{Cu}-\mathrm{MMC}(\mathrm{IR} 800)-\mathrm{TOC}$ than ${ }^{64} \mathrm{Cu}-\mathrm{DA}(\mathrm{IR} 800)$ TOC (5.2 \pm 0.2 vs. $3.6 \pm 0.4$ percentage injected dose per gram). In vivo blocking studies with octreotide reduced tumor uptake of ${ }^{64} \mathrm{Cu}$ MMC(IR800)-TOC by $66 \%$. Excretion of ${ }^{64} \mathrm{Cu}-\mathrm{MMC}(\mathrm{IR} 800)-\mathrm{TOC}$ was primarily through the liver and spleen whereas ${ }^{64} \mathrm{Cu}-\mathrm{DA}(\mathrm{IR} 800)$ TOC was cleared through the kidneys. Ex vivo analysis at $24 \mathrm{~h}$ confirmed PET/CT data by showing near-infrared fluorescence signal in tumors and a tumor-to-muscle ratio of $5.3 \pm 0.8$ as determined by $\gamma$-counting. Conclusion: The findings demonstrate that drug design affected receptor pharmacology and suggest that the MMC scaffold is a useful tool for the development of dual-labeled imaging agents.

Received Dec. 7, 2016; revision accepted May 26, 2017.

For correspondence or reprints contact: Ali Azhdarinia, The University of Texas Health Science Center at Houston, McGovern Medical School, Institute of Molecular Medicine, 1825 Pressler St., SRB 330C, Houston, TX 77030.

E-mail: ali.azhdarinia@uth.tmc.edu

Published online Jun. 1, 2017.

COPYRIGHT (C 2017 by the Society of Nuclear Medicine and Molecular Imaging.
Key Words: dual labeling; multimodality imaging; PET; NIRF; SSTR

J Nucl Med 2017; 58:1858-1864

DOI: 10.2967/jnumed.116.187971

\section{8}

urgery is the primary treatment option for most solid tumors and can be curative if all cancer cells are removed. Landmark clinical studies have shown that intraoperative imaging with fluorescent contrast agents resulted in more accurate tumor identification than current methods, which rely on visual and tactile cues, thus demonstrating the emerging role of fluorescence-guided surgery in cancer $(1,2)$. Despite these powerful implications, fluorescence-guided surgery can see only lesions that are already exposed to the field of view or are at a maximum of a few millimeters beneath the surface. This limitation could be overcome by introducing imaging agents that are dual-labeled with reporters for fluorescent and PET imaging. Although it can be argued that a cocktail administration of a radiotracer and its fluorescent counterpart will provide clinical utility similar to a dual-labeled analog, major limitations exist with this rationale. Importantly, validated fluorescent analogs of clinical radiotracers are not currently available and thus require ground-up development of a new chemical entity. This would entail quantification of tracer accumulation in tissues to determine biodistribution, which is routinely performed with radioactive compounds, but highly challenging with fluorescent agents given the attenuation of low-energy fluorescent photons in tissue. In addition, dyes and chelators have distinct chemical structures and discrepancies in size, which will result in the formation of conjugates with different physiochemical properties. This primarily impacts pharmacokinetics because chelators are polar compounds that facilitate renal clearance, whereas dyes are more hydrophobic and increase hepatobiliary clearance. As a result, the goal of obtaining signals from both reporters at the same time from the same location is highly challenging with separately administered contrast agents.

Conversely, a dual-labeled compound inherently provides temporal colocalization of signals. Moreover, the addition of a fluorescent dye to a clinical radiotracer directly expands utility from preoperative use to real-time intraoperative use. However, the conversion of clinical PET tracers (e.g., radiopeptides) into dual-labeled counterparts comes with significant technical challenges in agent design that occur from the attachment of largemolecular-weight fluorescent dyes to targeting agents of similar or 
even smaller size. Thus, novel strategies are needed to support the clinical translation of targeted multimodality imaging agents.

Somatostatin receptor (SSTR)-targeted peptides are widely used for imaging of neuroendocrine tumors (NETs) (3-8) and play an important role in determining appropriate treatment strategies. For several types of NETs, including the broad classes of pancreatic and gastric NETs (9), surgery is indicated not only for localized NETs, but also for advanced tumors to control excessive hormone production (10). In this regard, SSTR-targeted peptides would be ideal candidates for the development of multimodality counterparts because they would add intraoperative imaging utility to clinically validated imaging agents. Imaging could be performed during open surgery or with laparoscopic imaging systems (11) to enable better tumor visualization, identification of occult lesions, and margin detection. The most commonly used radiolabeled somatostatin analogs use the SSTR subtype 2 (SSTR2)-targeting peptides $\mathrm{Phe}^{1}-\mathrm{Tyr}^{3}$-octreotide (TOC) and $\mathrm{Phe}^{1}-\mathrm{Tyr}^{3}$-octreotate (TATE) for PET imaging with ${ }^{68} \mathrm{Ga}$ and ${ }^{64} \mathrm{Cu}(12-14)$. In addition to their use for pre- and postoperative imaging, radiolabeled somatostatin analogs have been used for radioguided surgery and have proven to be superior to preoperative imaging (scintigraphy and PET) and palpation for NET detection $(15,16)$. A key limitation of radioguided surgery, however, is the use of audible detection of signal, which does not provide surgeons with visual identification of contrast-enhanced lesions. Surgeons are, therefore, unable to use radioguided surgery to identify tumor margins and could still perform incomplete resections, which could increase the risk of local recurrence. Combining the proven imaging characteristics of radiolabeled somatostatin analogs with the higher spatial resolution of fluorescence imaging could overcome these limitations and further improve surgical outcomes by producing a dual-labeled somatostatin analog that complements existing intraoperative detection methods.

In this study, TOC was selected as a model for dual labeling to provide a proven targeting approach and benchmark the performance of a hybrid analog. Because the addition of a fluorescent dye onto DOTATOC could cause changes in pharmacology, specificity, and pharmacokinetics, we used a multimodality chelation (MMC) scaffold based on the macrocyclic compound 1,4,7,10-tetraazacyclododecane-1,7-diacetic acid (DO2A) to enable site-specific dye labeling and minimize steric effects. We compared the receptor agonist, binding, and internalization properties of duallabeled MMC-TOC with a more conventionally synthesized counterpart in SSTR2-expressing cell lines and performed multimodal imaging in a pancreatic acinar tumor-bearing mouse model (AR42J).

\section{MATERIALS AND METHODS}

All chemicals were purchased from Sigma-Aldrich unless otherwise noted. DO2A was purchased from Chematech. 1,4,7,10tetraazacyclododecane-1,4,7-tris ( $t$-butyl acetate) was purchased from Macrocyclics. IRDye 800-dibenzocyclooctyne (DBCO) was purchased from Li-COR Bioscience. ${ }^{1} \mathrm{H}$ NMR and ${ }^{13} \mathrm{C}$ NMR spectra were recorded at ambient temperature using 300- and 600-MHz IBM-Bruker Avance NMR spectrometers. Chemical shifts $(\delta)$ were reported (in ppm) downfield of tetramethylsilane. Reversed-phase high-performance liquid chromatography was performed on an analytic Hitachi LaChrom system using a Kinetex $\mathrm{C} 18$ column ( $2.6 \mu \mathrm{m}$; Phenomenex) with a mobile phase of $\mathrm{A}=0.1 \%$ trifluoroacetic acid in $\mathrm{H}_{2} \mathrm{O}, \mathrm{B}=0.1 \%$ trifluoroacetic acid in $\mathrm{CH}_{3} \mathrm{CN}$ (gradient: $0 \mathrm{~min}, 10 \% \mathrm{~B} ; 12 \mathrm{~min}, 90 \% \mathrm{~B}$ ), and a flow rate of $1 \mathrm{~mL} / \mathrm{min}$. Radiochemical purities of $95 \%$ or greater were assessed by radio-high-performance liquid chromatography (radio-HPLC) using an in-line radioactive detector (Berthold Technologies).

\section{${ }^{64} \mathrm{Cu}$ Radiolabeling}

The fluorescently labeled dual conjugate, 6 (herein referred to as MMC(IR800)-TOC), was synthesized according to Figure 1 (synthesis details are provided in the supplemental materials [available at http://jnm.snmjournals.org]). Radiolabeling was performed by mixing 1.5-20 nmol with $37-74 \mathrm{MBq}(1-2 \mathrm{mCi})$ of ${ }^{64} \mathrm{CuCl}_{2}$ (Washington University) in $0.1 \mathrm{M} \mathrm{NaOAc}$ (pH 6). Reactions were heated at $50^{\circ} \mathrm{C}$ for $1 \mathrm{~h}$, and radiochemical purity was monitored by radio-HPLC. Radiochemical purities were greater than $95 \%$ in all cases and did not require further purification.

\section{In Vitro Studies}

Pharmacologic properties of peptide conjugates were examined in HEK293 cells expressing HA3-tagged rsstr2, and methods are described in the supplemental materials. AR42J cells (American Type Culture Collection) were used for radioactive uptake studies and were cultured

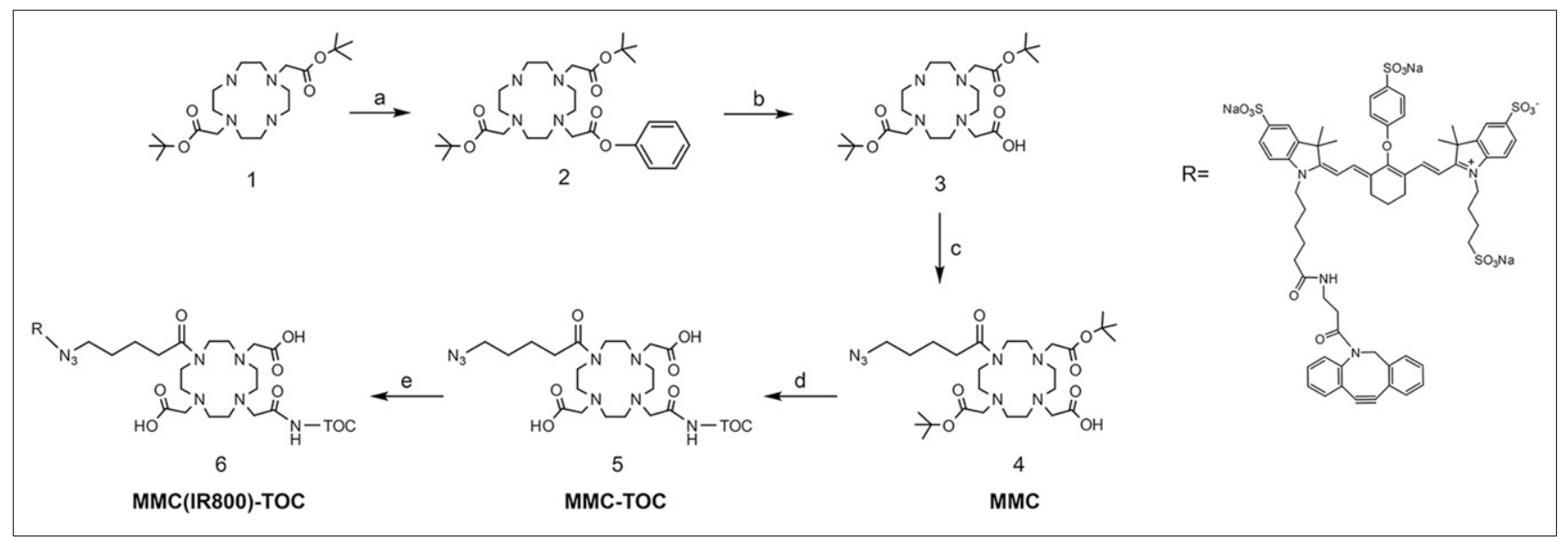

FIGURE 1. Synthesis of MMC(IR800)-TOC. a: Benzyl bromoacetate, $\mathrm{K}_{2} \mathrm{CO}_{3}, \mathrm{CH}_{2} \mathrm{Cl}_{2}$, r.t., $4 \mathrm{~h}$ stirring; b: $\mathrm{Pd} / \mathrm{C}$, cyclohexadiene, ethanol, $50^{\circ} \mathrm{C}, 5 \mathrm{~h}$ stirring; c: 5-azido-pentanoic acid, $\mathrm{N}$-(3-dimethylaminopropyl)- $\mathrm{N}$-ethylcarbodiimide hydrochloride (EDC), 1-hydroxybenzotriazole hydrate (HOBt), r.t., $4 \mathrm{~h}$ stirring, $\mathrm{N}, \mathrm{N}$-diisopropylethylamine (DIEA), $\mathrm{CH}_{2} \mathrm{Cl}_{2}$, r.t., overnight stirring; d: solid-phase peptide synthesis, trifluoroacetic acid (TFA) cleavage, oxidation; e: IRDye $800-\mathrm{DBCO}, \mathrm{DMSO} / \mathrm{H}_{2} \mathrm{O}(3: 1), 37^{\circ} \mathrm{C}, 4$ h, r.t., overnight stirring. $\mathrm{R}=\mathrm{IRDye} 800-\mathrm{DBCO}$. 


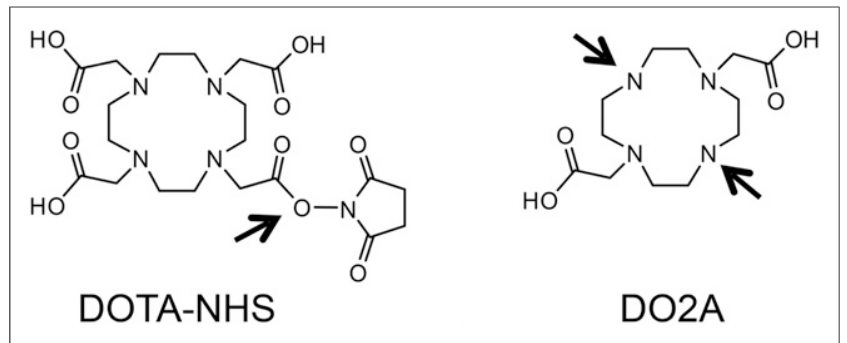

FIGURE 2. Chemical structures of macrocyclic chelators used for synthesizing peptide conjugates. Arrows designate reactive sites where selective conjugation of targeting agents or dyes can occur.

in $\mathrm{F}-12 \mathrm{~K}$ medium with $20 \%$ fetal bovine serum and $5 \% \mathrm{CO}_{2}$. Cells $(250,000)$ were added to each well of a 96 -well plate in serum-free RPMI followed by a $10 \mathrm{nM}(37-74 \mathrm{kBq})$ solution of the peptide conjugates. Incubation was performed at $37^{\circ} \mathrm{C}$ for $1 \mathrm{~h}$. For the blocking studies, cells were treated with 1 and $5 \mu \mathrm{M}$ octreotide (a 100- and 500fold excess over radiolabeled compound, respectively) immediately before addition of the tracers. At the end of the incubation period, the cells were pelleted and washed 3 times with phosphate-buffered saline. Cellbound radioactivity was quantitated in a Wizard ${ }^{2}$ automated $\gamma$-counter (Perkin Elmer) to determine uptake as percentage of total radioactivity added. Each experiment was performed in triplicate.

\section{In Vivo Imaging}

Animal studies were performed in accordance with the standards of the Institutional Animal Care and Use Committee of the University of Texas Health Science Center at Houston. Female 6- to 8-wk-old nu/nu mice were subcutaneously implanted with $3 \times 10^{6}$ AR42J cells with
Matrigel (1:1) in the left shoulder. Imaging studies were conducted 2-3 wk after implantation when tumor size reached approximately 5-10 $\mathrm{mm}$ maximum diameter. Mice $(n=3)$ were intravenously injected with $7.4 \mathrm{MBq}$ (200 $\mu \mathrm{Ci}, 2 \mathrm{nmol}$ ) of ${ }^{64} \mathrm{Cu}-\mathrm{MMC}(\mathrm{IR} 800)-\mathrm{TOC}$ or ${ }^{64} \mathrm{Cu}-\mathrm{DA}(\mathrm{IR} 800)-\mathrm{TOC}$, and imaging was performed at $4 \mathrm{~h}$ after injection on a Siemens Inveon small-animal PET/CT scanner as previously described (17). For blocking studies, mice were coinjected with ${ }^{64} \mathrm{Cu}-\mathrm{MMC}(\mathrm{IR} 800)$-TOC and a 10-fold excess of octreotide. For ${ }^{64} \mathrm{Cu}-\mathrm{MMC}(\mathrm{IR} 800)$-TOC, imaging was also performed at $24 \mathrm{~h}$ after injection $(n=5)$ to examine delayed imaging effects. Region-of-interest analysis was performed with the vendor software package (Inveon Research Workplace) to obtain tumor-to-background ratios. Near-infrared fluorescence (NIRF) images were acquired for $200 \mathrm{~ms}$ without background subtraction using a custom-built electron-multiplying charge-coupled device fluorescence imaging system at an excitation/ emission wavelength of $785 / 830 \mathrm{~nm}$ (18), and images were analyzed with the ImageJ software package. Parameters were the same for all acquired images. At the conclusion of the imaging studies, the mice were euthanized and selected organs were excised and underwent ex vivo NIRF imaging. Tissue radioactivity was quantified as previously described, and results were expressed as percentage injected dose (\% ID/g) (19).

\section{Statistical Analysis and Curve Fitting}

All data are presented as mean \pm SD as indicated. Means were compared using the Student $t$ test. The level of significance was set at a $P$ value of 0.05 . Nonlinear curve fitting was performed using the operational model in GraphPad Prism (version 7.0).

\section{RESULTS}

\section{Chemistry and Radiolabeling}

MMC(IR800)-TOC was synthesized using DO2A (Fig. 2) and obtained by conjugating IRDye $800-\mathrm{DBCO}$ to MMC-TOC.

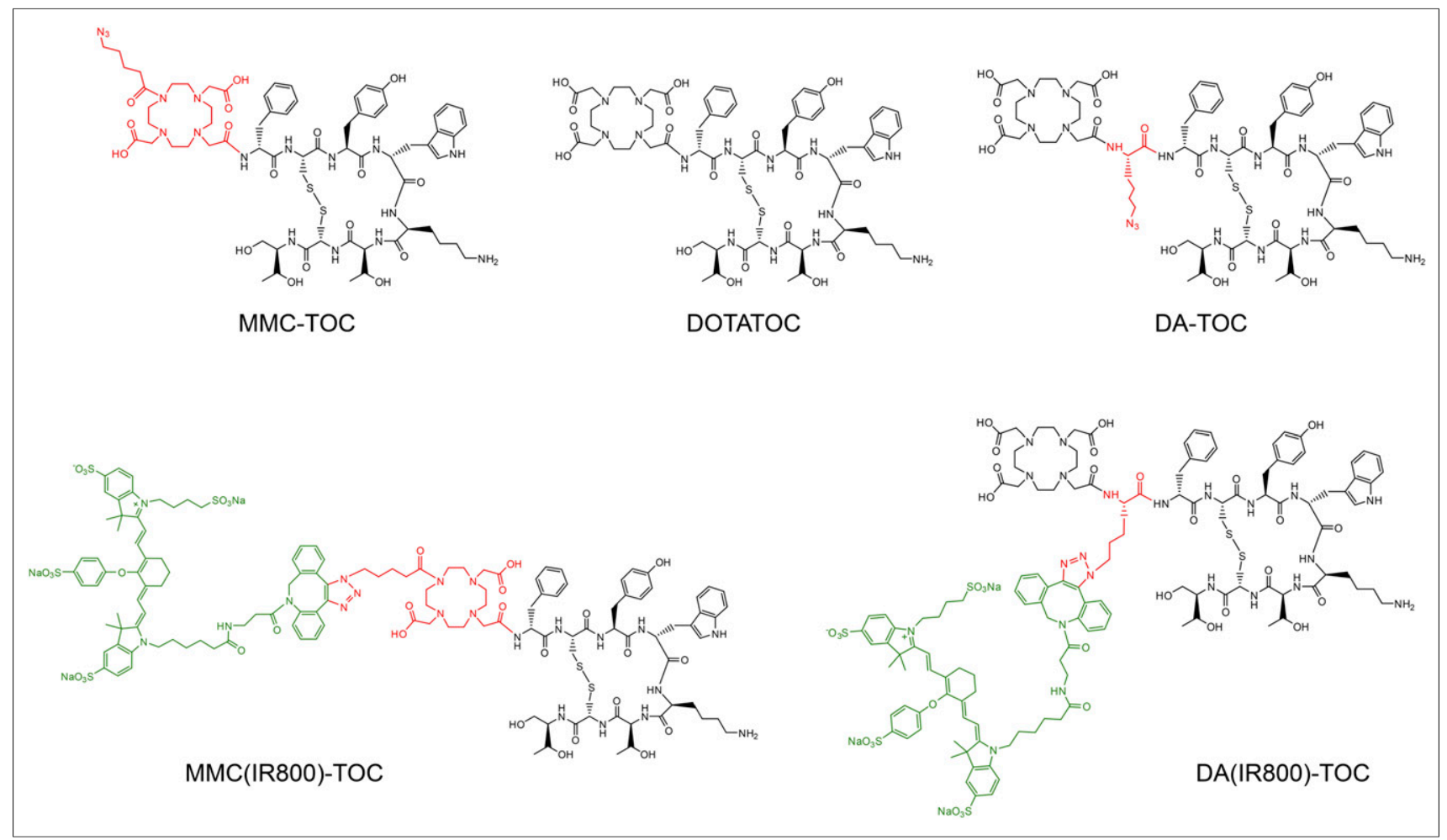

FIGURE 3. Chemical structures of DOTATOC and peptide conjugates using MMC and DA strategies. Red designates sites where chemical modifications were introduced. Dye moiety is displayed in green. 
TABLE 1

Radiochemical Stability in Mouse Serum

\begin{tabular}{cccc}
\hline & \multicolumn{4}{c}{ Incubation time } \\
\cline { 2 - 4 } Agent & $1 \mathrm{~h}$ & $2 \mathrm{~h}$ & $4 \mathrm{~h}$ \\
\hline${ }^{64} \mathrm{Cu}-$ DOTATOC & $99.4 \pm 0.3$ & $99.5 \pm 1.0$ & $98.4 \pm 0.9$ \\
${ }^{64} \mathrm{Cu}-\mathrm{MMC}($ IR800)-TOC & $97.3 \pm 1.0$ & $98.0 \pm 0.2$ & $98.4 \pm 0.3$
\end{tabular}

Data normalized to $100 \%$ at $\mathrm{t}=0$ and represent mean $\pm \mathrm{SD}(n=3)$.

Chemical structures of the peptide conjugates are shown in Figure 3 , and dual labeling was confirmed by reversed-phase HPLC (Supplemental Fig. 1). Labeling yields of $95 \%$ were achieved with $1.5 \mathrm{nmol}$ of each peptide and were greater than $98 \%$ with increasing peptide amounts. Specific activities as high as $49 \mathrm{TBq} / \mathrm{mmol}$ $(1,333 \mathrm{Ci} / \mathrm{mmol})$ were obtained. High radiochemical stability was observed for both ${ }^{64} \mathrm{Cu}$-DOTATOC and ${ }^{64} \mathrm{Cu}-\mathrm{MMC}(\mathrm{IR} 800)-$ TOC at 4 h serum incubation ( $>98 \%$ intact) (Table 1) (methods are described in the supplemental materials).

\section{In Vitro Pharmacology}

To determine of the effects of dye conjugation on agonist properties, we measured the potency of the dual-labeled compounds to inhibit cyclic adenosine monophosphate (cAMP) formation and to stimulate receptor internalization (methods are described in the supplemental materials). As shown in Figure 4A and Table 2, all compounds were able to maximally inhibit cAMP formation. Cu-DA-TOC was 4-fold less potent than Ga-DOTATOC. After dye conjugation, the half maximal effective concentration $\left(\mathrm{EC}_{50}\right)$ value of $\mathrm{Cu}-\mathrm{DA}(\mathrm{IR} 800)$-TOC increased and was 17-times less potent than the nonfluorescent precursor and 7-times less potent than the MMC counterpart. Results from the internalization studies (Fig. 4B; Table 2) demonstrated the efficacy of the MMC conjugate for agonist-stimulated receptor internalization. The $\mathrm{EC}_{50}$ value of $\mathrm{Cu}-\mathrm{MMC}(\mathrm{IR} 800)-\mathrm{TOC}$ was 10.9-fold lower than Cu-DA(IR800)-TOC.

\section{Cellular Uptake Assays}

As shown in Figure 5A, cellular uptake of ${ }^{64} \mathrm{Cu}-\mathrm{MMC}-\mathrm{TOC}$ and ${ }^{64} \mathrm{Cu}-\mathrm{MMC}(\mathrm{IR} 800)$-TOC in AR42J cells were similar and were reduced in the presence of a 100-fold molar excess of octreotide $(P<0.0001)$. By comparison, ${ }^{64} \mathrm{Cu}-\mathrm{DA}-\mathrm{TOC}$ had markedly lower uptake than its MMC counterpart $(22.1 \pm 1.5$ vs. $51.2 \% \pm 3.2 \%$, respectively), and dye labeling further reduced uptake to $4.8 \%$ for ${ }^{64} \mathrm{Cu}$-DA(IR800)-TOC (Fig. 5B). Although ${ }^{64} \mathrm{Cu}-\mathrm{DA}-\mathrm{TOC}$ uptake was readily blocked by octreotide, no differences in ${ }^{64} \mathrm{Cu}$-DA(IR800)-TOC uptake, and hence no specificity, were observed during competitive inhibition.

\section{Multimodality Imaging and Biodistribution}

Multimodality imaging studies were performed in mouse xenografts. As shown in Figure 6A, tumor uptake of ${ }^{64} \mathrm{Cu}-\mathrm{MMC}(\mathrm{IR} 800)-$ TOC was well visualized by PET/CT at $4 \mathrm{~h}$ and was higher than ${ }^{64} \mathrm{Cu}$-DA(IR800)-TOC. Blocking with octreotide reduced ${ }^{64} \mathrm{Cu}-\mathrm{MMC}(\mathrm{IR} 800)-\mathrm{TOC}$ signal in tumors, suggesting specificity of the MMC analog for SSTR2. ${ }^{64} \mathrm{Cu}-\mathrm{MMC}($ IR800)TOC showed high liver signal, indicating hepatobiliary clearance, whereas ${ }^{64} \mathrm{Cu}-\mathrm{DA}(\mathrm{IR} 800)-\mathrm{TOC}$ was primarily cleared through the kidneys. ${ }^{64} \mathrm{Cu}-\mathrm{MMC}(\mathrm{IR} 800)$-TOC signal was also seen in the gastrointestinal tract, along with uptake in the head region, which likely corresponds to physiologic uptake of the agent in salivary and pituitary glands (20).

Ex vivo biodistribution studies were conducted at $4 \mathrm{~h}$ after injection and are summarized in Figure 6B and Supplemental Figure 2. Tumor uptake was higher $(P<0.05)$ for ${ }^{64} \mathrm{Cu}-\mathrm{MMC}(\mathrm{IR} 800)$-TOC than ${ }^{64} \mathrm{Cu}-\mathrm{DA}(\mathrm{IR} 800)$-TOC $(5.23 \pm 0.16$ vs. $3.59 \pm 0.38 \% \mathrm{ID} / \mathrm{g}$, respectively), but tumor-to-muscle and tumor-to-blood ratios were similar because of lower background signal with ${ }^{64} \mathrm{Cu}-$ DA(IR800)-TOC. Analysis of the clearance organs showed higher uptake of ${ }^{64} \mathrm{Cu}-\mathrm{MMC}(\mathrm{IR} 800)-\mathrm{TOC}$ in reticuloendothelial system organs $(P<0.05)$ and a greater than 4-fold-higher uptake of ${ }^{64} \mathrm{Cu}$-DA(IR800)-TOC in the kidneys. Coinjection of octreotide resulted in a $66 \%$ reduction in tumor uptake of ${ }^{64} \mathrm{Cu}-\mathrm{MMC}(\mathrm{IR} 800)$ TOC compared with the unblocked group $(P<0.01)$. Delayed imaging and ex vivo biodistribution analysis of ${ }^{64} \mathrm{Cu}-\mathrm{MMC}(\mathrm{IR} 800)$ TOC at $24 \mathrm{~h}$ after injection revealed decreased tracer signal in most tissues, including tumors (Figs. 7A and 7B). In vivo NIRF imaging could not be conducted because the tumors had turned black at the time of imaging due to an unforeseen pigmentation effect (Supplemental Fig. 3). However, ex vivo NIRF imaging with ${ }^{64} \mathrm{Cu}-\mathrm{MMC}(\mathrm{IR} 800)$-TOC was in agreement with the PET/CT findings and showed tumor and liver uptake, as well as low muscle uptake (Fig. 7C).

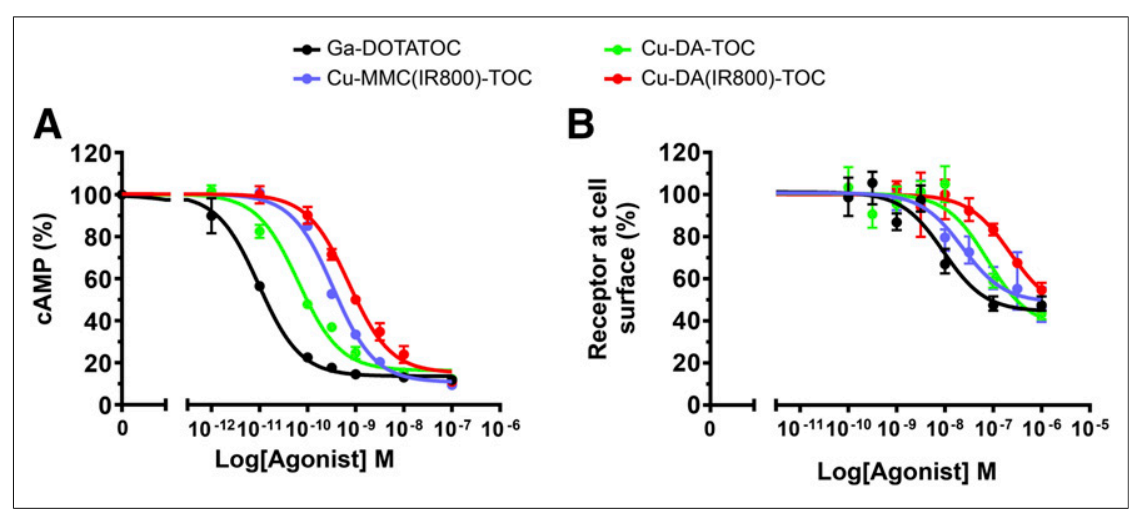

FIGURE 4. Characterization of agonist properties of peptide conjugates. (A) HEK293-HA3rsstr2-Glo cells were used to evaluate potency for cAMP inhibition. (B) HEK293-HA3-rsstr2 cells were used to measure potency of peptide conjugates to stimulate receptor internalization.

\section{DISCUSSION}

Several strategies have been proposed to facilitate the translation of intraoperative imaging agents, including the use of dual labeling (21). To maximize the likelihood of efficacy, a dual-labeled agent would ideally use a validated tumor-seeking agent, which can itself be used as a gold standard to assess radiolabeling efficiency, binding properties, and in vivo performance. To that end, we selected the somatostatin analog TOC as the model system in this study. TOC has been iteratively modified for radioiodination (22), radiometal chelation for $\gamma$-scintigraphy and PET $(7,23)$, and peptide receptor radionuclide therapy (24). Dual-labeled analogs of 
TABLE 2

Potencies and Efficacies of Peptide Conjugates

\begin{tabular}{lcc}
\hline & \multicolumn{2}{c}{$\mathrm{EC}_{50}(\mathrm{nM})$} \\
\cline { 2 - 3 } \multicolumn{1}{c}{ Agonist } & $\begin{array}{c}\text { cAMP } \\
\text { inhibition }\end{array}$ & $\begin{array}{c}\text { Receptor } \\
\text { internalization }\end{array}$ \\
\hline Ga-DOTATOC & $0.02 \pm 0.01$ & $11.0 \pm 0.8$ \\
\hline Cu-DA-TOC & $0.08 \pm 0.07$ & $78.3 \pm 37.7$ \\
Cu-DA(IR800)-TOC & $1.38 \pm 0.54$ & $455 \pm 299$ \\
\hline Cu-MMC(IR800)-TOC & $0.21 \pm 0.18$ & $41.9 \pm 29.8$
\end{tabular}

Data are expressed as mean \pm SD of the maximum possible effect for the agonist $\left(E_{\max }\right)$ values, which were calculated as difference between maximum and minimum signal for each analog and expressed as percentage of $E_{\max }$ value for internal control, Ga-DOTATOC.

TOC (25) and TATE (26) have also been tested preclinically and further indicate the robustness of this approach for contrast agent development. Most importantly, the clinical use of ${ }^{68} \mathrm{Ga}$ - and ${ }^{64} \mathrm{Cu}-$ labeled somatostatin analogs identify characteristics needed to obtain suitable contrast and can provide guidance for the development and optimization of dual-labeled TOC analogs.

Radiolabeled somatostatin analogs are generally synthesized by attaching a chelator to the $\mathrm{N}$ terminus of the peptide. Evidence from a recent study that used molecular modeling of a cross-bridged chelator-containing somatostatin analog revealed an unoccupied pocket next to the $\mathrm{N}$ terminus of receptor-bound $\mathrm{Tyr}^{3}$-TATE, supporting the rationale that substitution at this position should be well tolerated (27). This approach, however, proves significantly more challenging for a dual-labeled version of the peptide because commercial chelating agents generally possess 1 reactive site, which is occupied by peptide conjugation. As a result, the synthesis of dual-labeled peptides with conventional chelators and dyes necessitates alternative tactics (i.e., insertion of amino acids or spacers) that may adversely affect peptide binding. Synthesis of a dual-labeled somatostatin analog is further complicated by the need to avoid functionalization of the Lys-5 residue to retain receptor binding. Accordingly, we identified a bioorthogonal copper-free click chemistry approach with the MMC scaffold for site-specific and convenient dye conjugation. The scaffold is based on the macrocyclic compound DO2A because it contains 2 carboxylic acid moieties necessary for radiometal chelation, leaves 2 sites available for derivatization, and has the added advantage of maximizing the distance between attached $\mathrm{R}$ groups to reduce steric effects. Given the clinical efficacy of DOTA-based somatostatin analogs, we did not want to alter the DOTA-peptide configuration and instead focused on introducing modifications on the pendant arm of the MMC that is farthest removed from the peptide linkage site. Using this strategy, we employed the organic synthesis route described in Figure 1, which was well controlled, efficient, and scalable, to develop a MMC scaffold that is similar to DOTA in its macrocyclic core and chelation characteristics, but with added chemical versatility. This allowed us to selectively conjugate TOC and IRDye 800 to MMC at the 1 and 7 positions of the macrocycle, obtain high ${ }^{64} \mathrm{Cu}-$ labeling yields with as low as $1.5 \mathrm{nmol}$ of ligand and specific activity levels similar to non-dye-containing DOTA peptides (28), and eliminate the need to insert additional amino acids or spacers into the peptide sequence.

The efficacy of the MMC scaffold was shown by directly comparing MMC(IR800)-TOC with DA(IR800)-TOC, which possesses essentially the same chemical composition, to identify the impact of conjugation strategy on pharmacologic properties. In the potency assays (Fig. 4A; Table 2), Cu-DA-TOC effectively inhibited cAMP at levels that were comparable to Ga-DOTATOC $(0.08 \pm 0.03$ vs. $0.02 \pm 0.02 \mathrm{nM}$, respectively $)$. After dye conjugation, however, the potency of Cu-DA(IR800)-TOC was reduced by a factor of 17, and was 7 times lower than $\mathrm{Cu}-\mathrm{MMC}(\mathrm{IR} 800)-$ TOC. Similar results were observed in the receptor internalization studies (Fig. 4B; Table 2) and indicate that the proximity of the dye to the pharmacophore can significantly impair agonist potency. Radioactive uptake studies in AR42J cells were consistent with pharmacologic findings and showed no significant differences in the uptake of ${ }^{64} \mathrm{Cu}-\mathrm{MMC}-\mathrm{TOC}$ and ${ }^{64} \mathrm{Cu}-\mathrm{MMC}(\mathrm{IR} 800)-\mathrm{TOC}$, whereas the cellular uptake of ${ }^{64} \mathrm{Cu}-\mathrm{DA}-\mathrm{TOC}$ was reduced nearly 5-fold after dye conjugation (Figs. 5A and 5B). Receptor specificity of ${ }^{64} \mathrm{Cu}-\mathrm{DA}(\mathrm{IR} 800)$-TOC was also diminished by dye conjugation because blocking with octreotide did not reduce binding. This suggests significant nonspecific binding for ${ }^{64} \mathrm{Cu}-\mathrm{DA}($ IR800)TOC and is supported by the marked reduction in pharmacologic properties shown in Figure 4.

The in vivo properties of ${ }^{64} \mathrm{Cu}-\mathrm{MMC}(\mathrm{IR} 800)$-TOC were assessed in tumor-bearing mice by multimodality imaging. On $\mathrm{PET} / \mathrm{CT}$, tumors were clearly identified with ${ }^{64} \mathrm{Cu}-\mathrm{MMC}(\mathrm{IR} 800)$-TOC whereas lower tumor signal was found with ${ }^{64} \mathrm{Cu}-$ DA(IR800)-TOC. Blocking with octreotide significantly reduced the tumor uptake of ${ }^{64} \mathrm{Cu}-\mathrm{MMC}(\mathrm{IR} 800)-\mathrm{TOC}(P<0.01)$ and demonstrates SSTR2-mediated uptake. Consistent with ${ }^{64} \mathrm{Cu}$-DOTATATE biodistribution in patients (14), ${ }^{64} \mathrm{Cu}-\mathrm{MMC}(\mathrm{IR} 800)-\mathrm{TOC}$ uptake was observed in the liver (33.1 \pm $7.4 \% \mathrm{ID} / \mathrm{g})$ and spleen $(11.9 \pm 4.4 \% \mathrm{ID} / \mathrm{g})$ and indicates clearance through the reticuloendothelial system. The liver uptake could be attributed to a combination of hepatobiliary clearance and the kinetic stability of the cyclen-based chelation complex $(29,30)$. ${ }^{64} \mathrm{Cu}$-DA(IR800)-TOC had lower accumulation in reticuloendothelial system organs

in AR42J cells at $37^{\circ} \mathrm{C} .{ }^{\star \star *} P<0.0001$. 


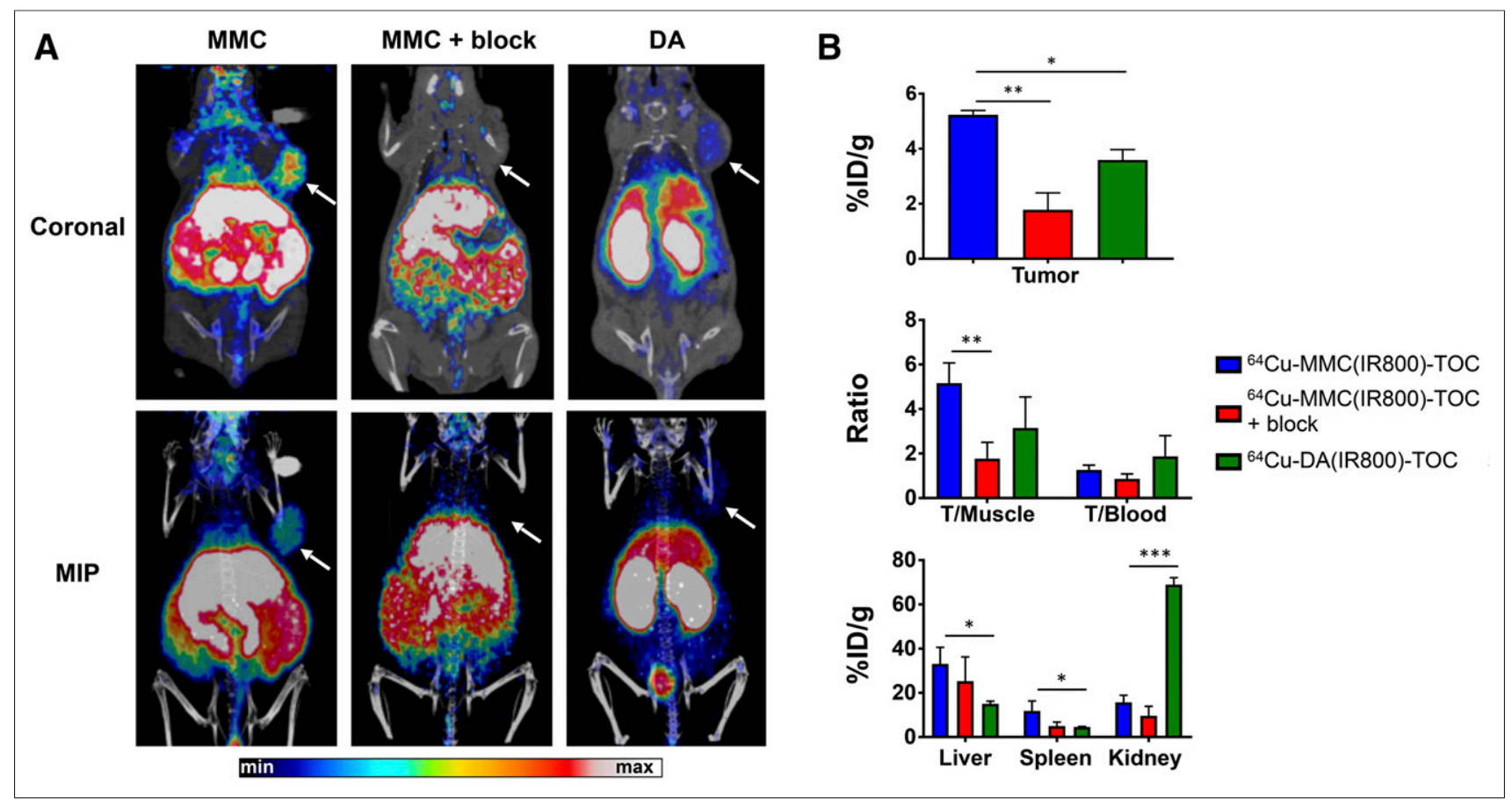

FIGURE 6. PET/CT imaging of AR42J xenografts $4 \mathrm{~h}$ after injection of ${ }^{64} \mathrm{Cu}-\mathrm{MMC}(\mathrm{IR} 800)-\mathrm{TOC}$ with and without blocking, and ${ }^{64} \mathrm{Cu}-\mathrm{DA}(\mathrm{IR} 800)-$ TOC. (A) Coronal images and maximum-intensity projections (MIP) are shown (arrow indicates tumor). (B) Ex vivo biodistribution analysis in AR42J xenografts injected with ${ }^{64} \mathrm{Cu}-\mathrm{MMC}(\mathrm{IR} 800)-\mathrm{TOC}$ with and without blocking, and ${ }^{64} \mathrm{Cu}-\mathrm{DA}(\mathrm{IR} 800)-\mathrm{TOC} .{ }^{*} P<0.05 .{ }^{* \star} P<0.01 .{ }^{* \star *} P<0.0001 . \mathrm{Max}=$ maximum; $\min =$ minimum; T/Blood $=$ tumor-to-blood ratio; T/Muscle $=$ tumor-to-muscle ratio.

and was primarily cleared through the kidneys $(69.0 \pm 3.2 \% \mathrm{ID} / \mathrm{g})$. The difference in clearance profiles may be due to the presence of an extra carboxylate that is present on DA, which could provide increased kinetic stability of the chelation complex in vivo while also increasing hydrophilicity. Notable ${ }^{64} \mathrm{Cu}-$ MMC(IR800)-TOC accumulation was also found in the intestines at $4 \mathrm{~h}$ after injection $(12.9 \pm 2.9 \% \mathrm{ID} / \mathrm{g})$ but decreased at $24 \mathrm{~h}$

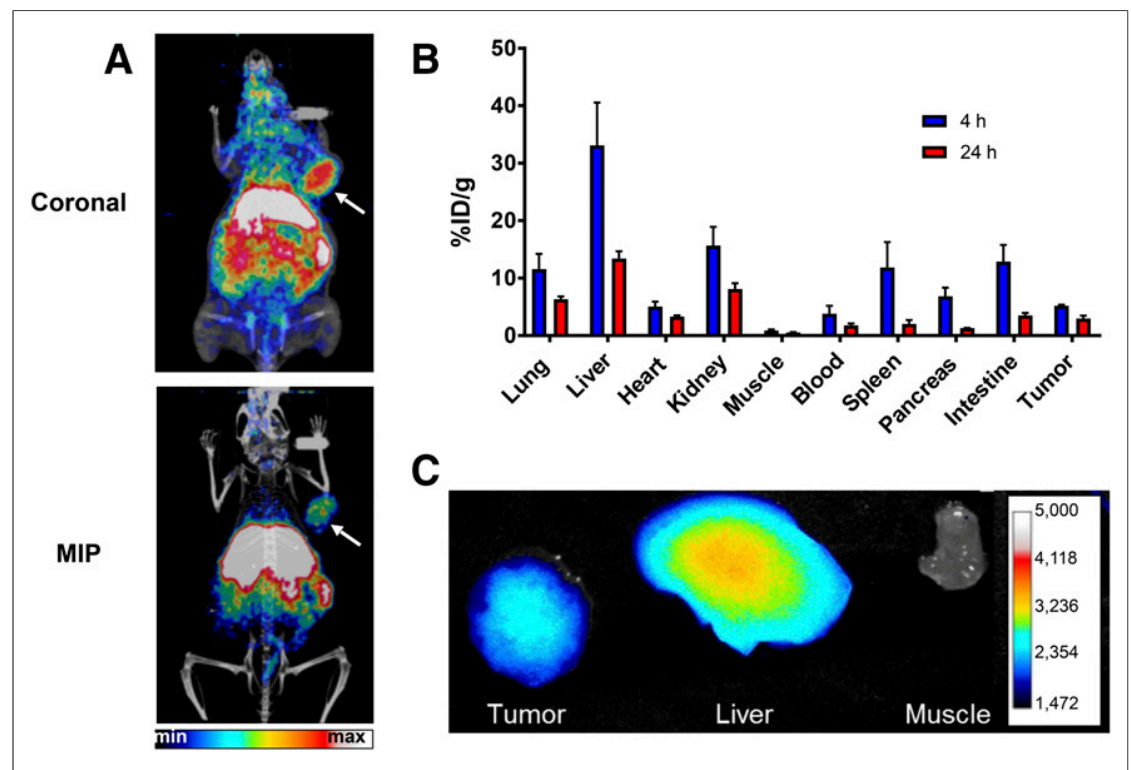

FIGURE 7. In vivo and ex vivo imaging of representative AR42J xenograft $24 \mathrm{~h}$ after injection of ${ }^{64} \mathrm{Cu}-\mathrm{MMC}$ (IR800)-TOC. (A) PET/CT images (arrow indicates tumor). Ex vivo analysis of tissues was performed by quantification of radioactivity with a y-counter (B) and NIRF imaging (C).
$(3.5 \pm 0.4 \% \mathrm{ID} / \mathrm{g})$ and is in agreement with preclinical data with ${ }^{64} \mathrm{Cu}$-DOTA-peptides $(31,32)$ and clinical data with ${ }^{64} \mathrm{Cu}$-DOTATATE (14). Although signal in nontumor tissues decreased over time (Fig. 7B), differences in tumor-to-nontumor ratios of ${ }^{64} \mathrm{Cu}-\mathrm{MMC}(\mathrm{IR} 800)-\mathrm{TOC}$ at 4 and $24 \mathrm{~h}$ were only significant in the pancreas $(P<0.005)$, suggesting that early imaging is sufficient for tumor visualization. It is conceivable that adding a carboxylic acid to the azide-containing pendant arm of the MMC would improve image contrast by combining the higher affinity and tumor targeting afforded by the MMC scaffold with the increased in vivo stability and low background signal shown by the DA conjugate. Alternatively, image contrast could be enhanced by developing MMC scaffolds with more kinetically inert ${ }^{64} \mathrm{Cu}$ chelators, such as 1,4,7-triazacyclononane,1glutaric acid-4,7-acetic acid (NODAGA) and 4,11-bis(carboxymethyl)-1,4,8,11tetraazabicyclo[6.6.2] hexadecane (CBTE2A) (33).

For in vivo NIRF imaging, an unexpected hyperpigmentation of AR42J tumors occurred at approximately 2 wk after implantation. To the best of our knowledge, this observation has not been reported for AR42J cells. Tumor pigmentation resulted in cold spots on the mouse images (Supplemental Fig. 3) but still displayed fluorescence signal ex vivo (Fig. 7C). Although AR42J tumors have played an important role in 
the preclinical characterization of radioactive imaging agents, our present study indicates that alternative tumor models may be better suited for evaluating SSTR2-targeted optical agents. Nonetheless, this study demonstrates the feasibility of the MMC scaffold for converting a clinically used radiotracer into a bioactive, multimodal analog, and encourages use with other peptide-based imaging agents.

\section{CONCLUSION}

A versatile dual-labeling method was developed in the present study to allow for integration into solid-phase peptide synthesis and accommodate biorthogonal chemistry approaches. We showed that drug design affected receptor pharmacology and that the MMC scaffold could successfully produce a multimodal agent that targets SSTR2-expressing cells and tumors.

\section{DISCLOSURE}

This work was supported by the National Institute of Biomedical Imaging and Bioengineering (NIBIB) (R01 EB017279), National Cancer Institute Network for Translational Research (NCI NTR) (U54 136404), and the Texas STAR Award Program. No other potential conflict of interest relevant to this article was reported.

\section{ACKNOWLEDGMENTS}

We thank Dr. Eva Sevick for technical discussions and access to NIRF imaging instrumentation, and Servando Hernandez Vargas and Randy Hickman for assistance with in vivo studies.

\section{REFERENCES}

1. Rosenthal EL, Warram JM, de Boer E, et al. Safety and tumor specificity of cetuximab-IRDye 800 for surgical navigation in head and neck cancer. Clin Cancer Res. 2015;21:3658-3666.

2. van Dam GM, Themelis G, Crane LM, et al. Intraoperative tumor-specific fluorescence imaging in ovarian cancer by folate receptor-alpha targeting: first inhuman results. Nat Med. 2011;17:1315-1319.

3. Reubi JC, Schonbrunn A. Illuminating somatostatin analog action at neuroendocrine tumor receptors. Trends Pharmacol Sci. 2013;34:676-688.

4. Rodrigues M, Traub-Weidinger T, Li S, Ibi B, Virgolini I. Comparison of ${ }^{111} \mathrm{In}$ DOTA-DPhe1-Tyr3-octreotide and ${ }^{111}$ In-DOTA-lanreotide scintigraphy and dosimetry in patients with neuroendocrine tumours. Eur J Nucl Med Mol Imaging. 2006;33:532-540.

5. Gabriel M, Muehllechner P, Decristoforo C, et al. ${ }^{99 \mathrm{~m} T c-E D D A} / \mathrm{HYNIC}-\mathrm{Tyr}(3)-$ octreotide for staging and follow-up of patients with neuroendocrine gastroentero-pancreatic tumors. Q J Nucl Med Mol Imaging. 2005;49:237-244.

6. Win Z, Al-Nahhas A, Towey D, et al. ${ }^{68} \mathrm{Ga}$-DOTATATE PET in neuroectodermal tumours: first experience. Nucl Med Commun. 2007;28:359-363.

7. Hofmann M, Maecke H, Borner R, et al. Biokinetics and imaging with the somatostatin receptor PET radioligand ${ }^{68} \mathrm{Ga}$-DOTATOC: preliminary data. Eur J Nucl Med. 2001;28:1751-1757.

8. Wild D, Macke HR, Waser B, et al. ${ }^{68} \mathrm{Ga}$-DOTANOC: a first compound for PET imaging with high affinity for somatostatin receptor subtypes 2 and 5. Eur J Nucl Med Mol Imaging. 2005;32:724.

9. Partelli S, Maurizi A, Tamburrino D, et al. GEP-NETS update: a review on surgery of gastro-entero-pancreatic neuroendocrine tumors. Eur J Endocrinol. 2014;171: R153-R162.

10. Birnbaum DJ, Turrini O, Vigano L, et al. Surgical management of advanced pancreatic neuroendocrine tumors: short-term and long-term results from an international multi-institutional study. Ann Surg Oncol. 2015;22:1000-1007.

11. van der Poel HG, Buckle T, Brouwer OR, Valdes Olmos RA, van Leeuwen FW. Intraoperative laparoscopic fluorescence guidance to the sentinel lymph node in prostate cancer patients: clinical proof of concept of an integrated functional imaging approach using a multimodal tracer. Eur Urol. 2011;60:826-833.

12. Kroiss A, Shulkin BL, Uprimny C, et al. ${ }^{68}$ Ga-DOTATOC PET/CT provides accurate tumour extent in patients with extraadrenal paraganglioma compared to ${ }^{123}$ I-MIBG SPECT/CT. Eur J Nucl Med Mol Imaging. 2015;42:33-41.

13. Menda Y, Ponto LL, Schultz MK, et al. Repeatability of gallium-68 DOTATOC positron emission tomographic imaging in neuroendocrine tumors. Pancreas. 2013;42:937-943.

14. Pfeifer A, Knigge U, Mortensen J, et al. Clinical PET of neuroendocrine tumors using ${ }^{64} \mathrm{Cu}$-DOTATATE: first-in-humans study. J Nucl Med. 2012;53: 1207-1215.

15. Adams S, Baum RP. Intraoperative use of gamma-detecting probes to localize neuroendocrine tumors. Q J Nucl Med. 2000;44:59-67.

16. Adams S, Baum RP, Hertel A, et al. Intraoperative gamma probe detection of neuroendocrine tumors. J Nucl Med. 1998;39:1155-1160.

17. Hall MA, Pinkston KL, Wilganowski N, et al. Comparison of mAbs targeting epithelial cell adhesion molecule for the detection of prostate cancer lymph node metastases with multimodal contrast agents: quantitative small-animal PET/CT and NIRF. J Nucl Med. 2012;53:1427-1437.

18. Hall MA, Kwon S, Robinson H, et al. Imaging prostate cancer lymph node metastases with a multimodality contrast agent. Prostate. 2012;72:129-146.

19. Ghosh SC, Ghosh P, Wilganowski N, et al. Multimodal chelation platform for near-infrared fluorescence/nuclear imaging. J Med Chem. 2013;56:406-416.

20. Virgolini I, Ambrosini V, Bomanji JB, et al. Procedure guidelines for PET/CT tumour imaging with ${ }^{68} \mathrm{Ga}$-DOTA-conjugated peptides: ${ }^{68} \mathrm{Ga}-\mathrm{DOTA}-\mathrm{TOC}$, ${ }^{68}$ Ga-DOTA-NOC, ${ }^{68}$ Ga-DOTA-TATE. Eur J Nucl Med Mol Imaging. 2010;37: 2004-2010.

21. Sevick-Muraca EM, Akers WJ, Joshi BP, et al. Advancing the translation of optical imaging agents for clinical imaging. Biomed Opt Express. 2013;4:160-170.

22. Krenning EP, Bakker WH, Breeman WA, et al. Localisation of endocrinerelated tumours with radioiodinated analogue of somatostatin. Lancet. 1989; $1: 242-244$.

23. Krenning EP, Bakker WH, Kooij PP, et al. Somatostatin receptor scintigraphy with indium-111-DTPA-D-Phe-1-octreotide in man: metabolism, dosimetry and comparison with iodine-123-Tyr-3-octreotide. J Nucl Med. 1992;33: $652-658$.

24. Kwekkeboom DJ, Mueller-Brand J, Paganelli G, et al. Overview of results of peptide receptor radionuclide therapy with 3 radiolabeled somatostatin analogs. J Nucl Med. 2005;46(suppl 1):62S-66S.

25. Santini C, Kuil J, Bunschoten A, et al. Evaluation of a fluorescent and radiolabeled hybrid somatostatin analog in vitro and in mice bearing H69 neuroendocrine xenografts. J Nucl Med. 2016;57:1289-1295.

26. Edwards WB, Xu B, Akers W, et al. Agonist-antagonist dilemma in molecular imaging: evaluation of a monomolecular multimodal imaging agent for the somatostatin receptor. Bioconjug Chem. 2008;19:192-200.

27. Cai Z, Ouyang Q, Zeng D, et al. ${ }^{64} \mathrm{Cu}$-labeled somatostatin analogues conjugated with cross-bridged phosphonate-based chelators via strain-promoted click chemistry for PET imaging: in silico through in vivo studies. $\mathrm{J} \mathrm{Med}$ Chem. 2014;57:6019-6029.

28. Roosenburg S, Laverman P, Joosten L, et al. PET and SPECT imaging of a radiolabeled minigastrin analogue conjugated with DOTA, NOTA, and NODAGA and labeled with ${ }^{64} \mathrm{Cu},{ }^{68} \mathrm{Ga}$, and ${ }^{111} \mathrm{In}$. Mol Pharm. 2014;11: 3930-3937.

29. Dearling JL, Voss SD, Dunning P, et al. Imaging cancer using PET: the effect of the bifunctional chelator on the biodistribution of a ${ }^{64} \mathrm{Cu}$-labeled antibody. Nucl Med Biol. 2011;38:29-38.

30. Ghosh SC, Pinkston KL, Robinson H, et al. Comparison of DOTA and NODAGA as chelators for ${ }^{64} \mathrm{Cu}$-labeled immunoconjugates. Nucl Med Biol. 2015;42:177-183.

31. Chen K, Ma W, Li G, et al. Synthesis and evaluation of ${ }^{64} \mathrm{Cu}$-labeled monomeric and dimeric NGR peptides for MicroPET imaging of CD13 receptor expression. Mol Pharm. 2013;10:417-427.

32. Persson M, El Ali HH, Binderup T, et al. Dosimetry of ${ }^{64} \mathrm{Cu}-\mathrm{DOTA}-\mathrm{AE} 105$, a PET tracer for uPAR imaging. Nucl Med Biol. 2014;41:290-295.

33. Fani M, Del Pozzo L, Abiraj K, et al. PET of somatostatin receptor-positive tumors using ${ }^{64} \mathrm{Cu}$ - and ${ }^{68} \mathrm{Ga}$-somatostatin antagonists: the chelate makes the difference. J Nucl Med. 2011;52:1110-1118. 